Rev. Elev. Méd. vét. Pays trop., 1979, 32 (1) : 33-35

\title{
Note sur un foyer de Chlamydiose abortive sur la chèvre au Tchad
}

\author{
par P. C. LEFEVRE $\left({ }^{*}\right)$, K. BAKETANA $\left({ }^{*}\right)$ et L. BERTAUDIERE $\left(^{*}\right)$
}

\section{RÉSUMÉ}

Une étude sérologique a été réalısée lors d'un foyer d'avortements de chèvres. La sérologie positive élevée vis-à-vis de l'antigène Chlamydia ovis, l'absence de brucellose et les commémoratifs incitent à penser qu'il s'agit de chlamydiose abortive dont la présence est décelée pour la première fois au Tchad.

\section{INTRODUCTION}

Les avortements des petits ruminants sont, en Afrique centrale, une dominante pathologique qu'il faudra maîtriser si une exploitation rationnelle de ce cheptel est envisagée. Des études réalisées en 1977 (1) donnent les taux de 28 p. 100 d'avortements par rapport aux mises bas et de 35 p. 100 rapporté aux femelles reproductrices.

De toutes les étiologies possibles, infectieuses ou non, les carences et les déséquilibres alimentaires sont, peut-on penser, les causes les plus fréquentes d'avortements. Toutefois, en l'absence de donnée précise concernant les infections abortives, la part des choses est difficile à faire.

Dans le but de préciser l'incidence des maladies infectieuses abortives, des enquêtes sérologiques ont été entreprises au Tchad.

Cet article rapporte l'existence vraisemblable d'avortements à Chlamydia ovis.

\section{MATÉRIEL ET MÉTHODES}

\section{A. Le foyer}

Une série d'avortements est apparue dans un troupeau de 153 chèvres comprenant 106 repro-

(*) Institut d'Elevage et de Médecine Vétérinarre des Pays Tropicaux. Laboratoire de Farcha, B. P. 433, N'Djaména, Tchad.

(**) Adresse actuelle : Projet F. A. O., B. P. 190, Gisenyi, République Rwandaise. ductrices au $1^{\text {er }}$ janvier 1977 (village de Gawi -région de N'Djaména). Entre le $1^{\text {er }}$ janvier et le

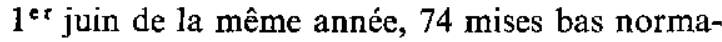
les ont été enregistrées et 19 avortements (uniquement en janvier, février, mars) ayant entraîné l'expulsion de 24 fotus morts. Des prises de sang ont été effectuées à l'époque pour la recherche de brucellose et un traitement à base de tétracycline instauré. Par la suite, les sérums ont été examinés par la technique de fixation du complément pour la recherche d'anticorps antichlamydiens.

\section{B. Prises de sang et commémoratifs}

Lors des prises de sang à la jugulaire, les commémoratifs (âge, sexe, nombre de mises bas, nombre d'avortements) ont été notés. Seuls les sérums de 106 animaux ( 86 brebis et 20 mâles) ont été récoltés.

C. L'étude sérologique pour le dépistage de la chlamydiose et de la brucellose a été réalisée à l'aide d'antigènes du commerce $(*)$ selon les techniques indiquées par les laboratoires producteurs. (Réaction de fixation de complément du type Kolmer à 2 unités de complément et fixation une nuit à $+4{ }^{\circ} \mathrm{C}$.)

(*) Laboratoire Roger BELLON : antigène de diagnostic des chlamydioses ovine et caprıne.

BIOMERIEUX : antigène brucellique pour la réaction de fixation du complément. 


\title{
III. RÉSUlTATS
}

Les résultats observés sont donnés dans les tableaux $n^{\circ}$ I et $n^{0}$ Il ci-après :

\begin{tabular}{|c|c|c|c|c|c|}
\hline Classe d'âge & Nombre par classe & Positif & p. 100 & Négatif & p. 100 \\
\hline \multirow{4}{*}{$\begin{array}{l}-1 \text { an } \\
1 \text { a } 2 \text { ans } \\
+2 \text { ans }\end{array}$} & 20 & 3 & 15 & 17 & 85 \\
\hline & 22 & 14 & 63,6 & 8 & 36,4 \\
\hline & 44 & 35 & 79,5 & 9 & 20,5 \\
\hline & 86 & 52 & 60,4 & 34 & 39,5 \\
\hline-1 an & 16 & 3 & - & 13 & - \\
\hline+1 an & 4 & 4 & - & 2 & - \\
\hline
\end{tabular}

\begin{abstract}
TABl.. N ${ }^{\circ}$ II-Pourcentage de femelles à sérologie chlamydienne positive ou négative selon les commémoratifs d'avortements.
\end{abstract}

\begin{tabular}{|c|c|c|c|c|}
\hline \multirow{2}{*}{ Positives } & Ayant \\
mis -bas & $\begin{array}{l}\text { Aydnt } \\
\text { avorte }\end{array}$ & $\begin{array}{c}\text { Par } \\
\text { rapport } \\
\text { au total }\end{array}$ & $\begin{array}{c}\text { Pourcentage } \\
\text { rapport aux } \\
\text { reproductions }\end{array}$ \\
\hline 52 & 38 & 16 & 30 & 42 \\
\hline Nêgatives & & & & \\
\hline 34 & 12 & 2 & 5,8 & 16,6 \\
\hline
\end{tabular}

\section{A. La chlamydiose}

Seuls les sérums présentant une hémolyse totale au $1 / 20$ et plus ont été considérés comme positifs. Un tiers des sérums sont positifs au $1 / 20$, un tiers au $1 / 40$, un quart au $1 / 80$ et le reste au $1 / 160$.

\section{B. La brucellose}

Sur 48 sérums analysés, 2 sont anticomplémentaires, 45 négatifs et 1 seul positif.

\section{DISCUSSION, CONCLUSION}

Le tableau I tend à faire admettre la progression de l'infection chlamydienne. Les jeunes sont en grande majorité négatifs (anticorps colostraux non décelés par la réaction, temps de contact relativement court...), alors qu'en revanche les trois quarts des femelles de plus de 2 ans sont trouvées porteuses d'anticorps.

Le tableau II souligne la relation qui existe entre le nombre d'avortements et la sérologie positive. Le test du $\chi^{2}$ est nettement significatif pour un risque d'erreur de 1 p. 100.

Il est bien évident que cette étude sérologique ne constitue pas une preuve de la présence d'avortements d'origine chlamydienne. Seul l'isolement de Chlamydia ovis d'un avorton ou du placenta peut apporter cette preuve.

Toutefois, dans ce foyer, la relation qui existe entre les avortements et une sérologie élevée vis-à-vis de la chlamydiose, l'absence de brucellose et les conditions d'entretien et d'alimentation satisfaisantes permettent de croire à l'existence de cette infection (2).

\section{SUMMARY}

Note on chlamydial abortions of goats in Chad

A serological study was realized after a period of abortions in a herd of goats. The results indicate that the abortions were due to Chlamydia ovis which is observed for the first time in Chad. 


\section{RESUMEN}

Nota sobre un foco de abortos a Chlamydia ovis en cabras en Chad.

Se realizó une encuesta serológica después de un foco de abortos en cabras. Los resultados indicán la presencia de Chlamydia ovis. Es la primera vez que se observó esta infección en Chad.

\section{BIBLIOGRAPHIE}

1. BERTAUDIÈRE (L.). Aspects de la reproduction et aptitude laitière chez la chèvre du Sahel (chèvre de Massakory). Rapport annuel. Laboratoire de Farcha. 1977.
2. DURAND (M.). Diagnostic des chlamydioses des ruminants : valeur de la fixation du complément. Rec. Méd. vét., 1977153 (9): 585-593. 